\title{
Power Control Policies for a Wireless Link with Energy Harvesting Transmitter and Receiver
}

\author{
Tingjun Chen, Sheng Zhou, Wei Chen, and Zhisheng Niu \\ Tsinghua National Laboratory for Information Science and Technology, \\ Dept. of Electronic Engineering, Tsinghua Univ., Beijing, 100084, P.R. China \\ Email: tjchen1203@gmail.com, \{sheng.zhou,wchen, niuzhs\}@tsinghua.edu.cn
}

\begin{abstract}
This paper addresses the outage minimization problem for a wireless link where both the transmitter and the receiver are powered by harvested energy, and the energy arrival processes of both nodes are correlated. We propose three power control policies to minimize the outage probability, including threshold-based On-Off policy, joint scheduling policy, and linear power levels policy. With infinite battery capacity, we analyze the optimality of the thresholds with different correlations between energy arrivals at the transmitter and the receiver. With finite battery capacity, we use finite state Markov chain (FSMC) to obtain the optimality of our policies and also numerically evaluate their performance. The optimal thresholds for minimum outages are derived according to the average energy arrival rate and the system parameters. The numerical results show the performance gains using different policies, as well as the tradeoff between the minimum outage probabilities and the average transmission times.
\end{abstract}

\section{INTRODUCTION}

This paper considers a wireless link with energy harvesting $(\mathrm{EH})$ transmitter and receiver. In such a scenario, harvested energy is injected into the link from the surrounding environment. Due to the randomness of the energy arrivals, the packet transmission between the link can fail when the energy stored in the link is not sufficient, and thus an outage occurs during the transmission. This motivates the need for new power control policies that adapt to the energy arrivals and power consumptions at both nodes of the link to achieve the minimum outage probability.

There has been some existing research about EH networks. When energy harvesting process is only considered at the transmitter, the throughput maximization problems and delay minimization problems are solved for singleuser cases. With infinite battery capacity, optimal power control policies are studied in [1] and [2]. With finite battery capacity, an optimal water-filling algorithm is developed in [3] and the causality of energy arrivals are also considered. Maximum stable throughput for $\mathrm{EH}$ cooperative networks are studied in [4] using stability analysis. To improve the reliability of the system, there has been recent research efforts on implementing the packet retransmission mechanism for energy harvesting communication systems. For example, ref. [5] models a system under an AQRbased retransmission mechanism and implements Markov Decision Processes (MDP) to obtain the optimal transmit power control policies. Ref. [6] also analyzes and evaluates the EH link performance using Markov evolution. However, none of these previous work has taken into consideration the energy consumption at the receiver which can be a key factor for packet transmission in realistic communication systems. To sustain the circuit blocks, as well as the decoding module, it is reasonable to consider the energy consumption at both the transmitter and the receiver. Additionally, we also need to consider the correlations between the energy arrival processes at both transceivers to analyze the dynamic evolution of the battery states.

In this paper, we focus on the outage minimization problem of a wireless link with energy harvesting transmitter and receiver. An acknowledgement (ACK) based retransmission mechanism is investigated. Power consumption are considered at both the transmitter and the receiver. We also consider the correlations between the energy harvesting profiles at both nodes and analyze the evolution of the battery states. We propose three transmit power control policies, including threshold-based On-Off policy, joint scheduling policy and linear power levels policy. With infinite battery capacity, we analyze the proposed power control policies and obtain the optimal thresholds under different scenarios. With finite battery capacity, the proposed policies are implemented based on a finite state Markov chain (FSMC) model, and their performances are evaluated numerically. For independent energy arrival process, we obtain the optimal threshold under the threshold-based On-Off policy. We analytically show that under the joint scheduling policy, the optimal transmit power is irrelevant to the correlation between the energy arrivals of both nodes. The value of the threshold is determined by the system parameters and the average energy arrival rate. The tradeoff between the average outage probability and the average transmission times are also shown numerically.

The rest of this paper is organized as follows. Section II sets up the system model, and three power control policies are proposed in Section III. In Section IV, we analyze the proposed policies and obtain the optimal thresholds for minimum outage probability with infinite battery capacity. We implement our policies with finite battery capacity using FSMC in Section V, and we propose the corresponding local searching algorithm for the optimal thresholds. Numerical results are presented in Section VI, and are followed by our conclusion in Section VII.

\section{SySTEM MODEL}

We consider a energy harvesting link consisting of one source node $S$ and one destination node $D$ which is shown in 


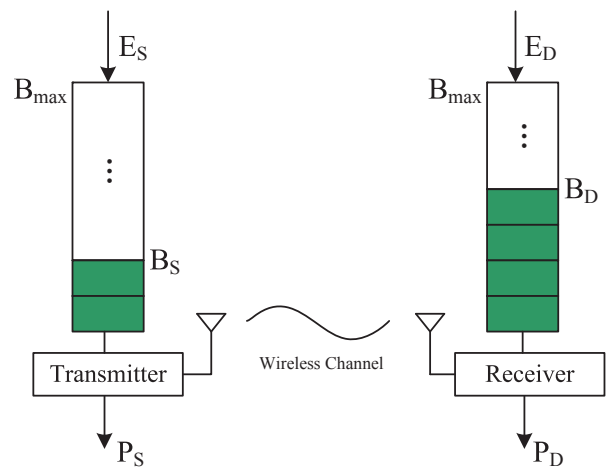

Fig. 1. System model for a wireless link with energy harvesting transmitter and receiver.

Fig. 1, both the nodes are powered by finite capacity energy batteries and are capable of harvesting energy from the surrounding environment. We focus on discrete-time model, where the time axis is partitioned into slots of unit duration, and we use $t \in\{0,1,2, \cdots\}$ to denote the indexes of the discrete time slots.

\section{A. The Energy Consumption Model}

To fully analyze the total energy consumption at the transmitter and the receiver, all signal processing blocks at the transmitter and the receiver need to be considered in the model. The model we used is according to [7]. The total power consumption at the transmitter and the receiver at slot $t$ are respectively given by

$$
\begin{aligned}
P_{S}^{t} & =(1+\alpha) P_{\mathrm{tx}}^{t}+P_{C, S}, \\
P_{D}^{t} & =P_{C, D},
\end{aligned}
$$

where $P_{C, S}$ and $P_{C, D}$ denote the static power consumption of the circuit blocks at $S$ and $D$. For a single link case, the average power consumption can be divided into two main components: the power consumption of all the power amplifiers $P_{P A}^{t}$ and the power consumption of all other circuit blocks $P_{C}$. It is shown [7] that the static energy consumption at $S$ is the sum of the power consumption for the block diagrams including Digital-to-Analog Converter (DAC), the mixer, the active filter at the transmitter side, and the frequency synthesizer. Similarly, the circuit energy consumption at $D$ is the sum of the power consumption values for the Low Noise Amplifier (LNA), the Intermediate Frequency Amplifier (IFA), the active filter, the Analogto-Digital Converter (ACD), and the frequency synthesizer. Besides the power used for the circuit blocks $P_{C}$, the power consumption for the power amplifiers $P_{P A}^{t}$ is a linear function of the actual transmitting power $P_{P A}^{t}=(1+\alpha) P_{\mathrm{tx}}^{t}$, where $\alpha=\frac{\xi}{\eta}-1$ with $\eta$ the drain efficiency and $\xi$ the Peakto-Average Ratio (PAR).

\section{B. The Energy Harvesting Model and Energy States Evolu- tion}

We consider two energy harvesting processes denoted as $\left\{E_{S}^{t}\right\},\left\{E_{D}^{t}\right\} \subset \mathbb{R}^{+}$which are independent sequences over the time axis but not independently distributed from each other. The variance of $\left\{E_{S}^{t}\right\}$ and $\left\{E_{D}^{t}\right\}$ is comparably small, and we assume $\mathbf{E}\left[E_{S}^{t}\right] \stackrel{\mathbf{E}}{=}\left[E_{D}^{t}\right]=\lambda_{e}$. Let $B_{S}^{t}$ and $B_{D}^{t}$ denote the battery states at the beginning of the $t$-th slot, and let $P_{S}^{t}$ and $P_{D}^{t}$ denote the power consumption strategy taken by the transmitter and the receiver, respectively. The battery gets replenished whenever a node harvests energy at the end of the slot. Thus, the energy evolution of the battery states is given by

$$
\left\{\begin{array}{l}
B_{S}^{t+1}=\min \left(B_{S}^{t}-P_{S}^{t}+E_{S}^{t}, B_{\max }\right), \\
B_{D}^{t+1}=\min \left(B_{D}^{t}-P_{D}^{t}+E_{D}^{t}, B_{\max }\right),
\end{array}\right.
$$

where $B_{\max }$ denotes the battery capacity and the indicator function $I(A)$ for the event $A$ is equal to one if $A$ occurs or else is zero. We also assume that $B_{S}^{0}=B_{D}^{0}=0$ for the initial battery states.

\section{The Channel Model and Outage Probability}

We consider stationary Rayleigh fading channel with frequency non-selective property, where the complex channel coefficient $h$ can be written as $h=X+j Y$ where $X$ and $Y$ are independent and identically distributed (i.i.d.) Gaussian random variables with zero mean and identical variance satisfying $\mathbf{E}\left(|h|^{2}\right)=1$. The probability density function (PDF) of $|h|$ is given by $f_{h}(r)=2 r \exp \left(-r^{2}\right)$, with $r=|h| \geq 0$. Thus the corresponding outage probability function is given by [8]

$$
\begin{aligned}
p\left(P_{\mathrm{tx}}\right) & =\operatorname{Pr}\left\{|h|^{2}<\frac{2^{R}-1}{P_{\mathrm{tx}}}\right\} \\
& =1-\exp \left(-\frac{2^{R}-1}{P_{\mathrm{tx}}}\right),
\end{aligned}
$$

where $R$ is the constant transmission rate which we assume is the same in all time slots, since the transmitter does not know the instantaneous channel state information (CSI). By defining $p(0) \triangleq 1$, we could see that $p\left(P_{\mathrm{tx}}\right)$ is strictly decreasing over its domain of $P_{\mathrm{tx}} \geq 0$.

Property 1: The outage probability function given in (4) is concave over $P_{\mathrm{tx}} \in\left[0, P_{b}\right]$ and convex over $P_{\mathrm{tx}} \in$ $\left[P_{b},+\infty\right)$, where $P_{b}=\frac{2^{R}-1}{2}$.

This non-convexity property can be easily derived by obtaining the second-order derivative of (4).

\section{The Packet Retransmission Scheme}

We consider the packet retransmission scheme as follows. At slot $t$, the packet transmission may fail if: (1) the transmitter does not have sufficient energy to transmit the packet $\left(B_{S}^{t}<P_{S}^{t}\right)$, (2) the packet is not transmitted successfully due to the outage occurs during the transmission with probability $p\left(P_{\mathrm{tx}}\right)$, (3) or the receiver does not have sufficient energy to receive the packet $\left(B_{D}^{t}<P_{D}^{t}\right)$. Thus, the probability that battery states of the link are sufficient to guarantee a successful transmission can be written as

$$
\Psi\left(P_{S}^{t}, P_{D}^{t}\right)=\lim _{n \rightarrow \infty} \frac{1}{n} \sum_{t=1}^{n} \mathbf{E}\left[I\left(B_{S}^{t} \geq P_{S}^{t}, B_{D}^{t} \geq P_{D}^{t}\right)\right]
$$




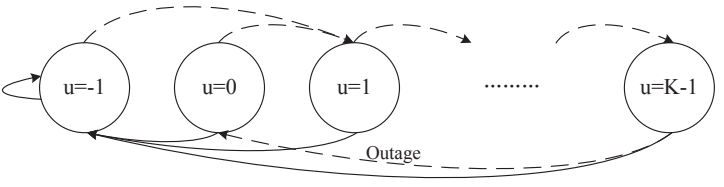

Fig. 2. Discrete time Markov evolution of ACK state $u$ under the retransmission scheme, where the dashed-lines represent a failed transmission, and the full lines represent a successful transmission. An outage occurs if and only if the ACK state is transformed for $u=K-1$ to $u=0$.

The retransmission process is based on an Acknowledgement and Negative-acknowledgement (ACK/NACK) based mechanism, in which short-length error-free packets are fed back by the destination $D$ over a separate narrow-band channel in order to inform the network about the packet reception status. If the packet is transmitted successfully, the transmitter will receive an ACK from the destination and the packet will be removed from the transmitter data queue. If the transmitter receives a NACK from the receiver, it retransmits its packet until it receives an ACK, or it runs out of energy, or it is time to drop the current packet due to the constraint of maximum retransmission times and to transmit the next packet. If it receives an ACK, the node will transmit the next packet immediately in the coming slot. We assume that the power consumed for sending ACK/NACK is negligible. Also, no CSI is assumed to be available at the transmitter.

The retransmission scheme is shown in Fig. 2. We allow $K-1$ times of retransmission ( $K$ times of transmission in total). Thus, the set of ACK states $\mathcal{U}$ is defined as

$$
\mathcal{U} \triangleq \begin{cases}-1, & \text { ACK received, } \\ 0, & \text { Start of a new transmission, } \\ u, & u \text {-th retransmission, } u \in\{1, \cdots, K-1\} .\end{cases}
$$

\section{E. Discussions of the Model}

Our model is focused on the behaviors of an energy harvesting link and energy consumptions are considered at both nodes of the link. At the transmitter, the power consumption of the circuit blocks can be regarded as a constant value $P_{C, S}$, but the total energy consumption $P_{S}^{t}$ is a linear function of the actual transmitting power $P_{\mathrm{tx}}^{t}$ shown in (1). At the receiver, we only consider the circuit power consumption $P_{D}^{t}=P_{C, D}$ as a constant value. However, this value can vary in different conditions [9]. For example, small $P_{C, D}$ values represent the receiver of an uncoded system where the power is only used for sustaining the components of the circuits blocks, while larger $P_{C, D}$ values represent the receiver of a coded system where the decoding power can not be ignored due to the complexity of the decoding processes.

\section{POWER Control Policies}

In this section, we present three power control policies based on the transmission index, battery energy levels, and the history of ACK/NACK messages received, to minimize the average outage probability.

\section{A. Threshold-based On-Off Policy}

Consider a one-threshold-based policy which refers to the simple On-Off policy, where the nodes do not know the energy state of each other and perform individually. Therefore, the sets of $P_{S}^{t}$ and $P_{D}^{t}$ are binary sets due to the On-Off policy where $P_{S}^{t} \in\left\{0, P_{S}\right\}$ and $P_{D}^{t} \in\left\{0, P_{D}\right\}$. Given the battery thresholds $P_{S}$ and $P_{D}$, the action will be made at each node if and only if its current battery state is equal or larger than the threshold at the beginning of each time slot. The battery states evolution under this policy is given by

$$
\left\{\begin{array}{l}
B_{S}^{t+1}=B_{S}^{t}-P_{S} I\left(B_{S}^{t} \geq P_{S}\right)+E_{S}^{t} \\
B_{D}^{t+1}=B_{D}^{t}-P_{D} I\left(B_{D}^{t} \geq P_{D}\right)+E_{D}^{t} .
\end{array}\right.
$$

\section{B. Joint Scheduling Policy}

Let us present a joint scheduling policy where the joint information of both the energy states at $S$ and $D$ are available at a control center. Thus, the battery states evolution under the joint scheduling policy is given by modifying (7) into:

$$
\begin{array}{r}
{\left[B_{S}^{t+1}, B_{D}^{t+1}\right]=\left[B_{S}^{t}, B_{D}^{t}\right]+\left[E_{S}^{t}, E_{D}^{t}\right]} \\
\quad-\left[P_{S}, P_{D}\right] I\left(B_{S}^{t} \geq P_{S}, B_{D}^{t} \geq P_{D}\right),
\end{array}
$$

where $\left[P_{S}^{t}, P_{D}^{t}\right] \in\left\{[0,0],\left[P_{S}, P_{D}\right]\right\}$ is the power consumption pair of the link. Here, the arithmetic function satisfies $\left[x_{1}, y_{1}\right] \pm\left[x_{2}, y_{2}\right]=\left[x_{1} \pm x_{2}, y_{1} \pm y_{2}\right]$. Under the joint scheduling policy, both the transmitter $S$ and the receiver $D$ consume a pair of non-zero power if and only if $B_{S}^{t} \geq P_{S}$ and $B_{D}^{t} \geq P_{D}$ hold at the same time.

\section{Linear Power Levels Policy}

We also consider a linear power levels policy denoted as an non-decreasing multi-level sequence $\left\{P_{S}(u)\right\}, u \in$ $\mathcal{U}$ with the same interval $\Delta_{P}$, and the respective total power consumption at the transmitter is given by $P_{S}^{t}(u)=$ $P_{S}(u) I\left(B_{S}^{t} \geq P_{S}(u)\right)$. This policy is a multi-levels transmitting policy that in $u$-th time of the transmission, if we have sufficient energy stored at the transmitter, we consume $P_{S}(u)$ and use $P_{\mathrm{tx}}(u)=\frac{P_{S}(u)-P_{C, S}}{1+\alpha}$ to transmit the packet. Otherwise, we do not transmit the packet and store the injected energy. We also assume that the transmitter does not have the battery information at the receiver. Thus, the battery states evolution under this policy is given by

$$
\left\{\begin{array}{l}
B_{S}^{t+1}=B_{S}^{t}-P_{S}(u) I\left(B_{S}^{t} \geq P_{S}(u)\right)+E_{S}^{t}, \\
B_{D}^{t+1}=B_{D}^{t}-P_{D} I\left(B_{D}^{t} \geq P_{D}\right)+E_{D}^{t},
\end{array}\right.
$$

where $u \in \mathcal{U}$ represents the retransmission index.

\section{OUtage ANALYsis With INFINIte Battery CAPACITY}

Obtaining the outage probability requires analyzing the probability $\Psi\left(P_{S}^{t}, P_{D}^{t}\right)$ defined in (5), which varies under different policies proposed before. In this section, we will analyze the outage probability in the case where both nodes are equipped with infinite battery capacities $\left(B_{\max } \rightarrow \infty\right)$. 


\section{A. Threshold-based On-Off Policy}

Under the Threshold-based On-Off policy, the battery states evolution is shown in (7). Each transmission during one slot is equivalent to each other since we consume a constant power $P_{\beta}(\beta=S, D)$ if the node $\beta$ has sufficient energy. Thus $P_{S}^{t} \in\left\{0, P_{S}\right\}$ and $P_{D}^{t} \in\left\{0, P_{D}\right\}$ are chosen from binary sets. The outage minimization problem (P1) can be given by maximizing the probability $\phi\left(P_{S}\right)$ that a transmission is successful, which is written as

$$
\text { (P1) } \max _{P_{S} \geq 0} \phi\left(P_{S}\right) \triangleq \exp \left(-\frac{2^{R}-1}{P_{\mathrm{tx}}}\right) \Psi\left(P_{S}, P_{D}\right) \text {. }
$$

The optimal solution of the threshold $P_{S}^{*}$ is given by

$$
P_{S}^{*}=\arg \max _{P_{S} \geq 0} \phi\left(P_{S}\right) .
$$

With $K$ times of transmission, in [12] we show that the ultimate outage probability is lower-bounded by

$$
P_{\text {out }}\left(P_{S}\right) \geq\left(1-\phi\left(P_{S}\right)\right)^{K} \text {. }
$$

From Eq. (12) we also show that $P_{S}^{*}$ derived by solving the maximization problem (P1) will also be the sub-optimal solution for the minimization problem for $P_{\text {out }}\left(P_{S}^{*}\right)$.

Proposition 1: Given independent energy arrivals $\left\{E_{S}^{t}\right\}$, $\left\{E_{D}^{t}\right\}$ and $B_{\max } \rightarrow \infty$, we have $\Psi\left(P_{S}, P_{D}\right)$ under the threshold-based On-Off policy given by

$$
\Psi\left(P_{S}, P_{D}\right)=\min \left(1, \frac{\lambda_{e}}{P_{S}}, \frac{\lambda_{e}}{P_{D}}, \frac{\lambda_{e}^{2}}{P_{S} P_{D}}\right),
$$

where $\mathbf{E}\left[E_{S}^{t}\right]=\mathbf{E}\left[E_{D}^{t}\right]=\lambda_{e}$.

Notice that $\left\{B_{S}^{t}\right\}$ and $\left\{B_{D}^{t}\right\}$ are independent, non-causal sequences, the proof of Proposition 1 can be obtained by extending the proof of [Theorem 1, 10] to the case of two independent battery state evolutions given by (7).

Remark 1: For non-independent energy arrivals $\left\{E_{S}^{t}\right\}$ and $\left\{E_{D}^{t}\right\}$, Proposition 1 still holds if $\lambda_{e}>\min \left(P_{S}, P_{D}\right)$. In this case, Proposition 1 becomes $\Psi\left(P_{S}, P_{D}\right)=$ $\min \left(1, \frac{\lambda_{e}}{P_{S}}, \frac{\lambda_{e}}{P_{D}}\right)$. Otherwise, if $\lambda_{e} \leq \min \left(P_{S}, P_{D}\right)$ which means that energy arrivals are insufficient at both nodes, it is expected that $\Psi\left(P_{S}, P_{D}\right)$ increases with $\rho$.

Theorem 1: Given independent energy arrivals, the optimal solution for the Problem (P1) under the threshold-based On-Off policy is given by

$$
P_{S}^{*}=\max \left(\lambda_{e}, B_{t h}\right),
$$

where $B_{t h}$ is a constant battery state threshold determined by $P_{C, S}, R$ and $\alpha$.

Proof: By applying (13) into (10), the optimal power consumption $P_{S}^{*}$ is obtained by deriving the first-order and second-order partial derivative of (10) over $P_{S}$ and is given by (14). The battery state threshold $B_{t h}=\frac{1}{2}\left(2 P_{C, S}+C_{R, \alpha}+\right.$ $\left.\sqrt{C_{R, \alpha}\left(C_{R, \alpha}+4\right)}\right)$, in which $C_{R, \alpha}=\left(2^{R}-1\right)(1+\alpha)$ is a constant value determined by $R$ and $\alpha$. In solution (14), $P_{S}^{*}=B_{t h}$ holds for the case $P_{S}=B_{t h} \geq \lambda_{e}$, and $P_{S}^{*}=$ $\lambda_{e}$ holds for both cases $P_{S} \geq \lambda_{e}, B_{t h}<\lambda_{e}$ and $P_{S}<$ $\lambda_{e}$. Thus, the optimal transmit power is given by $P_{\mathrm{tx}}^{*}=$ $\frac{1}{1+\alpha}\left(\max \left(\lambda_{e}, B_{t h}\right)-P_{C, S}\right)$.
The solution shown in (14) are both sufficient and necessary conditions of optimality for Problem (P1) under the threshold-based On-Off policy. The above proposition shows that when the energy arrivals are independent random processes at both nodes, the optimal transmit power $P_{\mathrm{tx}}^{*}$ at the transmitter is independent of the energy consumption $P_{D}$ at the receiver $D$, and it is only determined by its energy injection and system parameters, for example $P_{C, S}, \alpha$ and the transmission rate $R$.

\section{B. Joint Scheduling Policy}

Under the joint scheduling policy, the battery states evolution is shown in (8). To solve Problem (P1), we first present the following proposition.

Proposition 2: Given the energy arrivals $\left\{E_{S}^{t}\right\}$ and $\left\{E_{D}^{t}\right\}$ and $B_{\max } \rightarrow \infty$, we have $\Psi\left(P_{S}, P_{D}\right)$ under the joint scheduling policy given by

$$
\Psi\left(P_{S}, P_{D}\right)=\min \left(1, \frac{\lambda_{e}}{P_{S}}, \frac{\lambda_{e}}{P_{D}}\right) .
$$

The proof of Proposition 2 is provided in Appendix A. Using Proposition 2, we have the theorem shown below.

Theorem 2: Given two energy arrivals $\left\{E_{S}^{t}\right\}$ and $\left\{E_{D}^{t}\right\}$ with the same mean value $\lambda_{e}$, the optimal solution for the Problem (P1) under the joint scheduling policy is given by

$$
P_{S}^{*}=\max \left(\lambda_{e}, B_{t h}, P_{D}\right),
$$

where $B_{t h}$ is defined in Theorem 1.

Proof: Under the joint scheduling policy, both nodes know the battery state of each other and the transmitter transmits regarding the energy information of the receiver. Thus, the link consumes the power pair $\left[P_{S}, P_{D}\right]$ if and only if energy states are sufficient at both nodes. The optimal $P_{S}^{*}$ shown in (16) is given by solving the same optimization problem in (10) by applying (15).

Remark 2: From Proposition 2 and Theorem 2, we can see that the joint scheduling policy can smooth the correlation between $\left\{E_{S}^{t}\right\}$ and $\left\{E_{D}^{t}\right\}$. Given infinite battery capacity, the optimal transmit power $P_{\mathrm{tx}}^{*}$ is determined only by $\lambda_{e}, B_{t h}$ and $P_{D}$, regardless of the correlations between the two energy arrival processes.

\section{Linear Power Levels Policy}

Under the linear power levels policy, the battery states evolution is shown in (9). Given the intervals of the sequence $\left\{P_{S}(u)\right\}$ and $K$, we need to find the optimal starting power level $P_{S}(u=-1,0)$ to determine $\left\{P_{S}(u)\right\}$. We have the following proposition under the linear power levels policy.

Proposition 3: We use $\bar{\phi}$ given by

$$
\bar{\phi} \triangleq\left(1-\phi\left(P_{S}(u=0,-1)\right)\right) \prod_{u=1}^{K-1}\left(1-\phi\left(P_{S}(u)\right)\right)
$$

to approximate the ultimate outage probability as (12). Let $P_{t h}^{*}$ denotes the optimal power consumption given by (14) and $P_{l p}^{*}$ denotes the optimal starting power of $\left\{P_{S}(u)\right\}$, 
respectively. Given independent $\left\{E_{S}^{t}\right\}$ and $\left\{E_{D}^{t}\right\}$, for a nondecreasing linear power levels policy $\left\{P_{S}(u)\right\}$ we have

$$
P_{l p}^{*} \leq P_{t h}^{*}=\max \left(\lambda_{e}, B_{t h}\right) .
$$

Proof: Under the linear power levels policy, the transmit power $P_{\mathrm{tx}}$ of each slot is not independent with each other, and is corresponded with the ACK state index $u$. We denote $\bar{\phi}^{u} \triangleq 1-\phi\left(P_{S}(u)\right)$. From (17) we have $\frac{\partial \bar{\phi}}{\partial P_{S}}=$ $\frac{1}{\phi} \sum_{u} \bar{\phi}^{u} \frac{\partial \bar{\phi}^{u}}{\partial P_{S}}$. Notice that $\left\{P_{S}(u)\right\}$ is non-decreasing over the retransmission index $u$ and $\left.\frac{\partial \bar{\phi}^{u}}{\partial P_{S}}\right|_{P_{S}=P_{t h}^{*}}$ equals to 0 when $u=0,-1$ since it is the first time of a transmission. In the case $P_{S}<\lambda_{e}$ we have $\frac{\partial \bar{\phi}^{u}}{\partial P_{S}} \geq 0$ holds, and in the case $P_{S} \geq \lambda_{e}$ we can get $\left.\frac{\partial \bar{\phi}^{u}}{\partial P_{S}}\right|_{P_{S}=P_{t h}^{*}} \geq 0$, therefore $\left.\frac{\partial \bar{\phi}}{\partial P_{S}}\right|_{P_{t h}^{*}} \geq 0$ holds. It can be shown that there exists an optimal value $P_{l p}^{*} \leq P_{t h}^{*}$ satisfying $\left.\frac{\partial \bar{\phi}}{\partial P_{S}}\right|_{P_{S}=P_{l p}^{*}}=0$, and we complete the proof.

It can be shown from Proposition 3 that under the linear power levels policy, a heuristic way of choosing $P_{l p}^{*}$ of $\left\{P_{S}(u)\right\}$ should be no larger than $P_{t h}^{*}$ which is the optimal power consumption in the On-Off policy described in the previous subsection.

\section{OUtage Analysis with Finite Battery CAPACITY}

In this section, we discuss the case where both nodes are equipped with finite battery capacity. We implement our model using finite state Markov chain (FSMC), in which we normalize all energies with respect to a minimum possible fraction of energy consumption $E^{1}$. Thus, the battery levels are considered to be integers multiple of $E$.

\section{A. FSMC Formulation}

The discrete-time FSMC has the state space $\mathcal{S} \triangleq \mathcal{B}_{S} \times$ $\mathcal{B}_{D} \times \mathcal{U}$, where $\mathcal{B}_{\beta} \triangleq\left\{0,1, \cdots, B_{\max }\right\}(\beta=S, D)$ is the set of battery states of the node $\beta$. We also have $B_{\max }<\infty$. Recall that $\mathcal{U}=\{-1,0,1, \cdots, K-1\}$ is the set of possible packet transmission attempt states and the represented received ACK states. Thus, the state of the link at time $t$ is denoted by $s^{t} \triangleq\left(b_{S}^{t}, b_{D}^{t}, u^{t}\right)$ where $b_{\beta}^{t} \in \mathcal{B}_{\beta}$ and $u^{t} \in \mathcal{U}$.

For the energy harvesting processes, an i.i.d. Bernoulli model $\left\{E_{S}^{t}, E_{D}^{t}\right\}$ is considered. At the end of slot $t, E_{S}^{t}$ and $E_{D}^{t}$ levels of energy are injected into the transmitter and the receiver with probability $\mu_{S}$ and $\mu_{D}$, respectively. With probability $1-\mu_{S}$ and $1-\mu_{D}$, no energy is harvested. Similar model is also used in [5]. Assume the Bernoulli energy arrivals of the link are with same maximum value $E_{\max }$ and same mean value $\lambda_{e}$, this assumption is practical in the small scale networks, i.e., the wireless sensor networks (WSN). We also consider the correlation of the two energy arrivals using the correlation coefficient denoted as $\rho=\frac{\operatorname{cov}\left(E_{S}^{t}, E_{D}^{t}\right)}{\sigma_{E_{S}^{t}} \sigma_{E_{D}^{t}}^{t}}$

\footnotetext{
${ }^{1}$ We use the unit Joule/slot in our discretized model with the unit duration of a slot, where the conception of energy and power are quantized by the same value $E$ Joule/slot.
}

$(0 \leq \rho \leq 1)$ where $\operatorname{cov}(\cdot)$ is the covariance function, $\sigma_{E_{S}^{t}}$ and $\sigma_{E_{D}^{t}}$ represent the standard variances of $E_{S}^{t}$ and $E_{D}^{t}$.

Therefore, the harvested energy pair injected in to the link at slot $t$ can be written as

$$
\left(E_{S}^{t}, E_{D}^{t}\right)= \begin{cases}(0,0), & \text { with probability } \mu_{0} \\ \left(0, E_{\max }\right), & \text { with probability } \mu_{1} \\ \left(E_{\max }, 0\right), & \text { with probability } \mu_{2} \\ \left(E_{\max }, E_{\max }\right), & \text { with probability } \mu_{3}\end{cases}
$$

where $t=0,1,2, \cdots$. Each of the probabilities $\mu_{i}(i=$ $0,1,2,3)$ can be calculated through the previous definition of $\rho$ with the constrains: (1) $\sum_{i=0}^{i=3} \mu_{i}=1$ and (2): $\mu_{1}=\mu_{2}$.

We denote the state transition probability matrix as $\mathcal{G}$, whose elements represent the probability of a transition from state $s^{t} \triangleq(m, n, v)$ to state $s^{t+1} \triangleq(i, j, u)$. It is defined as

$$
\begin{array}{r}
\mathcal{G}_{m n v}^{i j u}=\operatorname{Pr}\left\{B_{S}^{t+1}=i, B_{D}^{t+1}=j, u^{t+1}=u\right. \\
\left.\mid B_{S}^{t}=m, B_{D}^{t}=n, u^{t}=v\right\},
\end{array}
$$

where $m, i \in \mathcal{B}_{S}, n, j \in \mathcal{B}_{D}$ and $u, v \in \mathcal{U}$. Let $\delta\left(s^{t}, a^{t}(u), s^{t+1}\right)$ denotes the Kronecker function from state $s^{t}$ to $s^{t+1}$ with an action $a^{t}(u)$ taken at slot $t$, with retransmission index $u$. The action $a^{t}(u)=\left[P_{S}^{t}(u), P_{D}^{t}\right]$ will vary corresponding to different power control policies. For $\forall v$ and $u=-1, \mathcal{G}_{s^{t}}^{s^{t+1}}=\mu\left(1-p\left(P_{\mathrm{tx}}\right)\right) \delta\left(s^{t}, a^{t}(u), s^{t+1}\right)$ if $a^{t}(u)=\left[P_{S}(u), P_{D}\right]$. For $v=K-1$ and $u=0$, or $v=-1$ and $u=1$, or $v \neq-1, K-1$ and $u=v+1$, $\mathcal{G}_{s^{t}}^{s^{t+1}}=\mu p\left(P_{\mathrm{tx}}\right) \delta\left(s^{t}, a^{t}(u), s^{t+1}\right)$ if $a^{t}=\left[P_{S}(u), P_{D}\right]$. For $v=K-1$ and $u=0$, or $v=-1$ and $u=1$, or $v \neq-1, K-1$ and $u=v+1, \mathcal{G}_{s^{t}}^{s^{t+1}}=\mu \delta\left(s^{t}, a^{t}(u), s^{t+1}\right)$ if $a^{t} \neq\left[P_{S}(u), P_{D}\right]$. Under the threshold-based On-Off policy and joint scheduling policy, we have $\left\{P_{S}(u)\right\}=P_{S}$ is the constant power consumption.

The stationary probabilities $\pi(s)$ of this FSMC can be obtained by solving the balance equation

$$
\pi\left(s^{t+1}\right)=\sum_{s} \operatorname{Pr}\left\{s^{t+1}=(i, j, u) \mid s^{t}=(m, n, v)\right\} \pi\left(s^{t}\right) .
$$

Under the ACK-based retransmission mechanism, an outage occurs if and only if a packet is still not transmitted successfully even after $K-1$ times of retransmission. We focus on the following problem of minimizing the average outage probability

$$
\min _{P_{S}^{t} \geq 0} P_{\text {out }},
$$

where the average outage probability $P_{\text {out }}$ is shown in the following proposition.

Proposition 4: The average outage probability of the link $S \rightarrow D$ is given by

$$
P_{\text {out }}=\frac{\sum_{u=0} \pi(s)}{\sum_{u=0} \pi(s)+\sum_{u=-1} \pi(s)},
$$

where $\pi(s)$ is the stationary probability of state $s$.

Proof: Notice that $\pi_{u=0}(s)$ represents a transmission outage occurs in the previous slot, and $\pi_{u=-1}(s)$ represents a successful transmission is finished in the previous slot. Otherwise, in the states $s$ where $u \neq-1,0$, the packet will be under retransmission in the current slot. Assuming 
we have $N$ slots and $N \rightarrow \infty$ to reach the stationary distribution, it can be obtained that the number of packets transmitted successfully is $N_{\text {success }}=N \sum_{u=-1} \pi(s)$, and the number of packets transmitted with an outage is $N_{\text {outage }}=N \sum_{u=0} \pi(s)$. Thus, the average outage probability is given by the ratio of the packets that are not transmitted successfully even after $K-1$ times of retransmission:

$$
P_{\text {out }}=\frac{N_{\text {outage }}}{N_{\text {outage }}+N_{\text {success }}},
$$

and we can get Proposition 4.

Similarly, the average transmission times $\tau$ is given by a weighed function of the stationary probability $\pi(s)$ :

$$
\tau=1+\frac{\sum_{k-1}^{K-1} k p_{-1, k} \pi_{v=k}(s)}{\pi_{u=-1}(s)},
$$

where $p_{u, v}$ is defined as $p_{u, v} \triangleq \sum_{(i, j)(m, n)} \operatorname{Pr}\left\{s^{t+1}=\right.$ $\left.(i, j, u) \mid s^{t}=(m, n, v)\right\}$.

We can now summarize the procedure for computing the outage probability $P_{\text {out }}$ and the average transmission times $\tau$ :

1) Discretize the batter levels and the power consumption levels to generate the FSMC with state space $\mathcal{S}$,

2) Compute $\mathcal{G}$, where $\mathcal{G}$ is the state transition probability matrix with entries define in (20),

3) Obtain the stationary probabilities $\pi(s)$ by solving (21),

4) Obtain $P_{\text {out }}$ and $\tau$ from (23) and (25), respectively.

Remark 3: In the infinite battery capacity case where $B_{\max } \rightarrow \infty$, it is obtained that if $\max \left(P_{S}, P_{D}\right)<\lambda_{e}$, the battery states will have the behaviors $B_{\beta} \rightarrow \infty(\beta=S, D)$. Thus the state space will become a simpler set denoted as $\mathcal{S}_{U}=\mathcal{U}=\{-1,0,1, \cdots K-1\}$. The corresponding transition matrix $\mathcal{G}_{U}$ is of dimension $K+1$ and the stationary distribution can be easily obtained as

$$
\pi_{U}=\frac{1-p}{1-p^{K}}\left[1, p^{K}, p, p^{2}, \cdots, p^{K-1}\right],
$$

where $p=p\left(P_{\mathrm{tx}}\right)$. Through (26), $P_{\text {out }}=p^{K}$ (which means $K$ times of transmission fail continuously) where $p$ is obtained through (4). However, in the cases where the battery capacity is bounded by $B_{\max }$, it is hard to get the closed-form of the stationary distribution $\pi(s)$.

\section{B. Local Searching Algorithm}

For the threshold-based On-Off policy and the joint scheduling policy requires carefully choosing the threshold $P_{S} \in \mathcal{B}_{S}$. For the linear power levels policy, a local searching algorithm is also implemented to find the optimal starting point $P_{S}^{*}=P_{S}^{-1}=P_{S}^{0}$ at a new transmission. Given $K$ and the intervals of the sequence $\left\{P_{S}(u)\right\}$, the transmit power levels is determined by $P_{S}^{*}$. A one-dimension local searching algorithm shown in Algorithm 1 is implemented to find the optimal $P_{S}^{*}$ and $P_{\mathrm{tx}}^{*}$. This algorithm requires searching all the states of the energy storage at the transmitter and is of complexity $\mathcal{O}\left(B_{\max }+1\right)$. We also have the transition matrix $\mathcal{G} \in \mathbb{R}^{\left(B_{\max }+1\right)^{2} \times(K+1)}$, and $\mathcal{G}$ will be different under different power control policies.
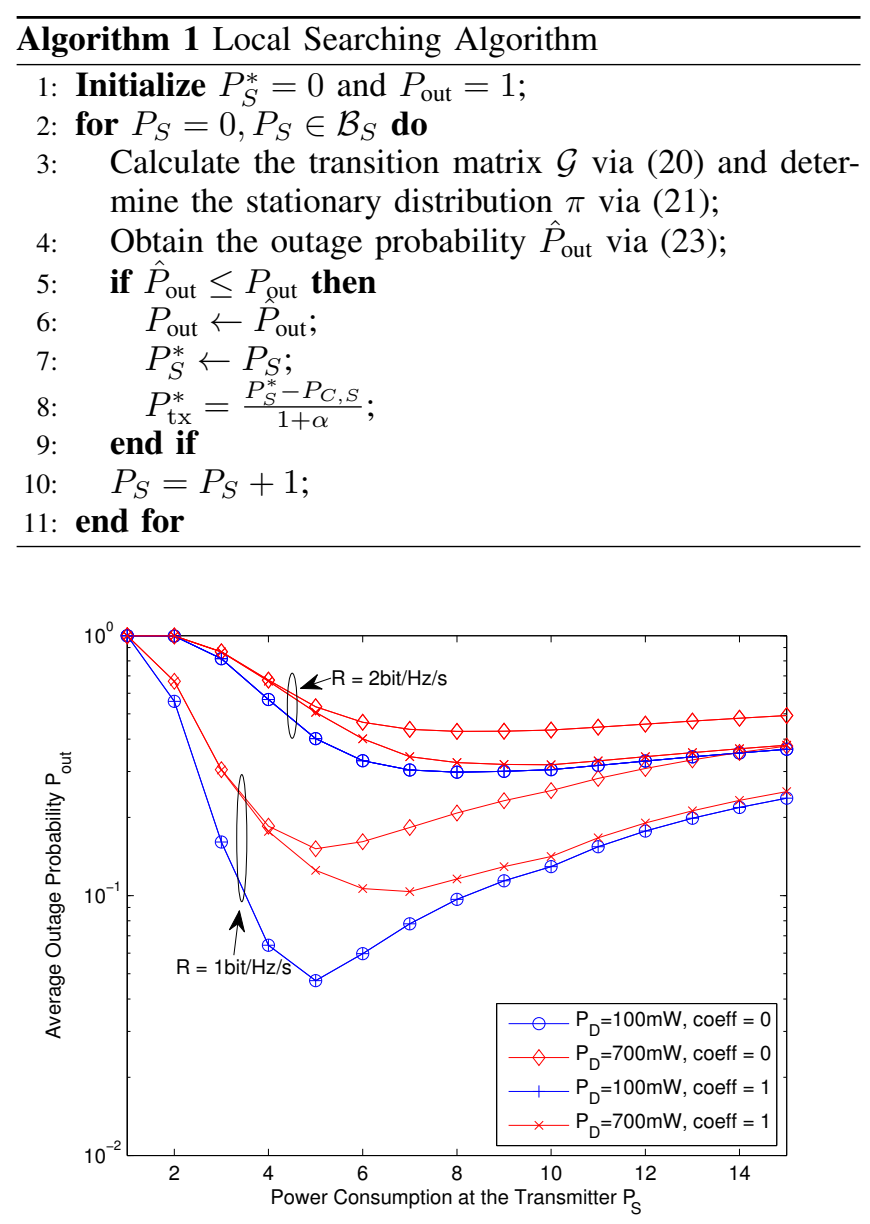

Fig. 3. Average outage probability under threshold-based On-Off policy, for $R \in\{1,2\} \mathrm{bit} / \mathrm{Hz} / \mathrm{s}, P_{D} \in\{100,700\} \mathrm{mW}$, and $\rho \in\{0,1\}$.

\section{NUMERICAL RESULTS}

In this section, we present numerical results to show the performance of the proposed power control policies. For normalization, the state space is discretized by the quantization granularity of $E=100 \mathrm{~mW}$, thus the energy and power are of the same quantization $E=100 \mathrm{~mW}$ over the same unit slot period. We consider the Bernoulli energy input profiles are with parameters $E_{\max }=10 E$ and $\lambda_{e}=5 E$, and $P_{C, S}=E$ is the constant circuits consumption at the transmitter. Given the correlation coefficient $\rho$, we can obtain $\mu_{i}(i=0,1,2,3)$ from (19). The battery has the finite capacity $B_{\max }=3 E_{\max }$. For the uncoded system where the decoding power is ignorable at the receiver, we let $P_{C, D}=$ $E=100 \mathrm{~mW}$ [7], otherwise a power of $P_{C, D}=700 \mathrm{~mW}$ is consumed at the receiver for decoding in the coded system [9]. The drain efficiency $\eta$ equals to 0.5 for a typical class$\mathrm{AB}$ amplifier [11] and thus $\alpha \approx 1$. Thus, $P_{S}^{t}=2 P_{\mathrm{tx}}^{t}+P_{C, S}$. For the retransmission mechanism, we allow $K=4$ times of transmission (3 times of retransmission), thus the ACK state set is $\mathcal{U}=\{-1,0,1,2,3\}$.

For the threshold-based On-Off policy, different transmission rates $R=1 \mathrm{bit} / \mathrm{Hz} / \mathrm{s}$ and $R=2 \mathrm{bit} / \mathrm{Hz} / \mathrm{s}$ are considered to satisfy all the conditions. In the case with infinite 


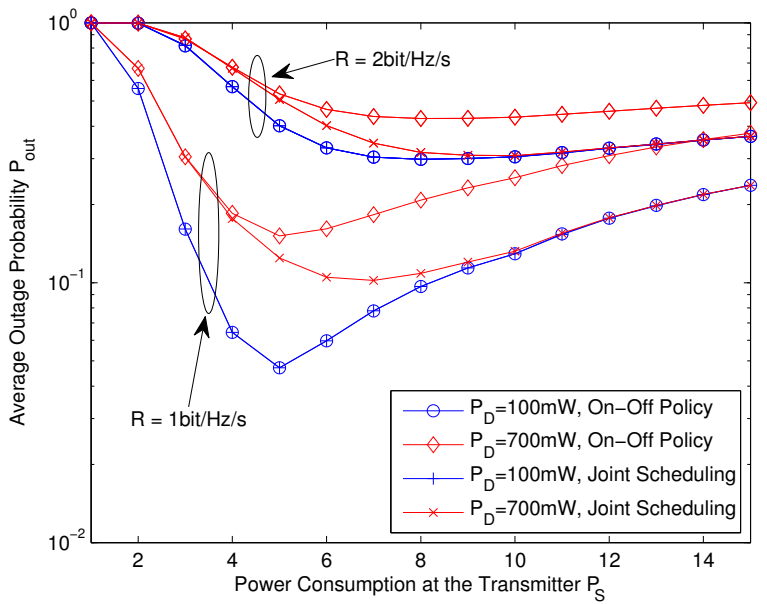

Fig. 4. Average outage probability under threshold-based On-Off policy and the joint scheduling policy, where $\rho=0$ for threshold-based On-Off policy and $\rho=0.5$ for joint scheduling policy.

battery capacity and independent energy arrivals $\rho=0$, if $R=2 \mathrm{bit} / \mathrm{Hz} / \mathrm{s}$ we have $P_{S}^{*}=B_{t h}=7.87 E>\lambda_{e}$ from (14). Also, if $R=1 \mathrm{bit} / \mathrm{Hz} / \mathrm{s}$ we have $P_{S}^{*}=B_{t h}=3.73 E<\lambda_{e}$. In the case with finite battery capacity, the average outage probabilities based on FSMC is shown in Fig. 3. With independent energy arrival processes $\rho=0$, we can see $P_{S}^{*}=\lambda_{e}=5 E$ for the case where $R=1 \mathrm{bit} / \mathrm{Hz} / \mathrm{s}$ (for sufficient energy input $\lambda_{e}>B_{t h}=3.73 E$ ), and $P_{S}^{*}=8 E$ in the case $R=1 \mathrm{bit} / \mathrm{Hz} / \mathrm{s}$ (for insufficient energy input $\lambda_{e}<B_{t h}=7.87 E$ ). This result shows that the local searching algorithm fits our analysis of the outage minimization problem (P1) where $B_{\max } \rightarrow \infty$. Thus, by calculating the energy arriving threshold $B_{t h}$ and compare it with $\lambda_{e}$, we could easily get the optimal transmit power threshold $P_{S}^{*}$ to achieve minimum outage probability with guaranteed performance. In the case with the same energy input processes $\rho=1$, the optimal $P_{S}^{*}=P_{D}$ when $P_{D}=7 E>\lambda_{e}$ and $B_{t h}<\lambda_{e}$. When $B_{t h}>\lambda_{e}$ with high transmission rate $R=2 \mathrm{bit} / \mathrm{Hz} / \mathrm{s}, P_{S}^{*}=8 E$ holds. Also, the average outage probability curves with $\rho=1$ is always lower than the curves with $\rho=0$. From the result, given constant transmit power $P_{\mathrm{tx}}=\frac{P_{S}-P_{C, S}}{1+\alpha}$, we can get lower outage probability in the cases where $P_{C, D}$, or the transmission rate $R$ is smaller, or the energy arrivals are sufficient. With larger energy consumption $P_{C, D}$ at the receiver, the outage probability will be affected by the correlation of the energy arrivals, which can be explained by (13).

We then show the numerical results of joint scheduling policy and compare it with the threshold-based OnOff policy. The average outage probability is shown in Fig. 4 with $\rho=0.5$ in the joint scheduling policy. For arbitrary $0 \leq \rho \leq 1$, the results are the same which confirm Proposition 2. It is shown that the joint scheduling policy outperforms the threshold-based On-Off policy. This is because that if the states of both nodes are known to each other and the link performs jointly, the system can achieve lower outage probability. Under this policy, $P_{S}^{*}=\lambda_{e}$ holds

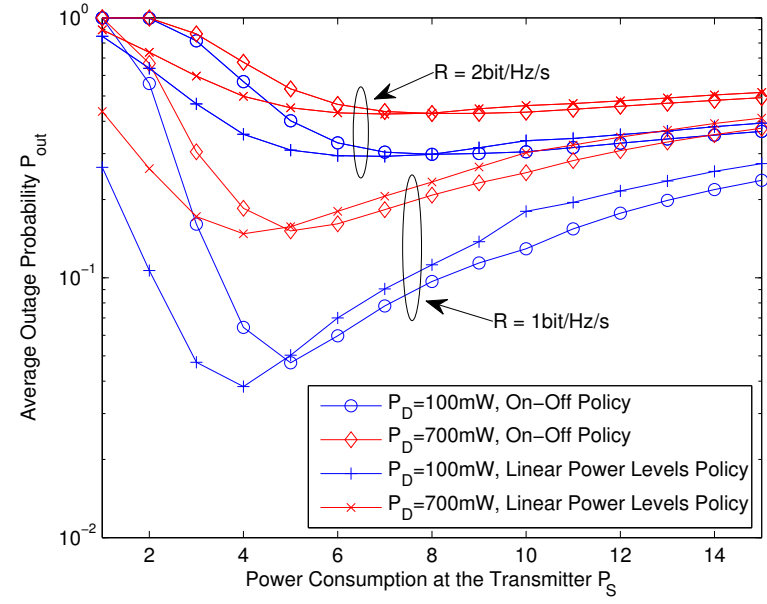

Fig. 5. Average outage probability under the threshold-based On-Off policy and linear power levels policy, for $R \in\{1,2\} \mathrm{bit} / \mathrm{Hz} / \mathrm{s}, P_{D} \in$ $\{100,700\} \mathrm{mW}$, and $\rho=0$.

when $R=1 \mathrm{bit} / \mathrm{Hz} / \mathrm{s}$ and $P_{D}<\lambda_{e} . P_{S}^{*}=P_{D}$ holds when $B_{t h}<\lambda_{e}$ with low $R$ and $P_{D}$. For $B_{t h}>\lambda_{e}$, we have $P_{S}^{*}=\max \left(B_{t h}, P_{D}\right)$ holds which confirms Theorem 2 .

For the linear power levels policy, we implement our method using the increasing power levels sequence with the same increasing value $\Delta=2 E$, that is $\left\{P_{S}(u)\right\}=$ $\left\{P_{S}, P_{S}+\Delta, P_{S}+2 \Delta, P_{S}+3 \Delta\right\}$. For $u=-1$ and $u=0$, $P_{S}^{-1}=P_{S}^{0}=P_{S}$. For $u \geq 1, P_{S}(u)=P_{S}+u \Delta$. We show the performance of the linear power levels policy compared with the previous threshold-based policy in Fig. 4, where we use $\rho=0$ to represent relatively independent energy arrivals. In Fig. 5, we can see that the linear power levels policy achieves a global minimum average outage probability lower than the threshold-based On-Off policy. The starting point of the linear power sequence $P_{l p}^{*}$ is lower than the optimal power consumption $P_{t h}^{*}$ obtained before. With lower $P_{S}$, the linear policy reaches a gain in the outage probability, while in the larger areas of $P_{S}$, the two curves become closer because of the insufficiency of the energy arrivals.

Finally, we show the tradeoff between the average outage probability $P_{\text {out }}$ and the average transmission time $\tau$ in Fig. 6 . We change the number of total transmission times $K$ represented at the horizontal axis. The four points on each curve represent the minimum outage probability $P_{\text {out }}$ and the corresponding average transmission times $\tau$. As it is shown in the Fig. 6, when we have higher $K, P_{\text {out }}$ will go down. From the curves, the threshold-based On-Off policy with $\rho=1$ at both nodes performs nearly same with the joint scheduling policy. With larger power consumption $P_{D}$ at the receiver,the linear power levels policy performs with the lowest outage. With small $P_{D}$ and $R$, linear power levels policy achieves the lowest outage probability with the cost of higher average transmission times.

\section{CONCLUSION}

We proposed three power control policies for the energy harvesting link and solved the outage minimization problem 


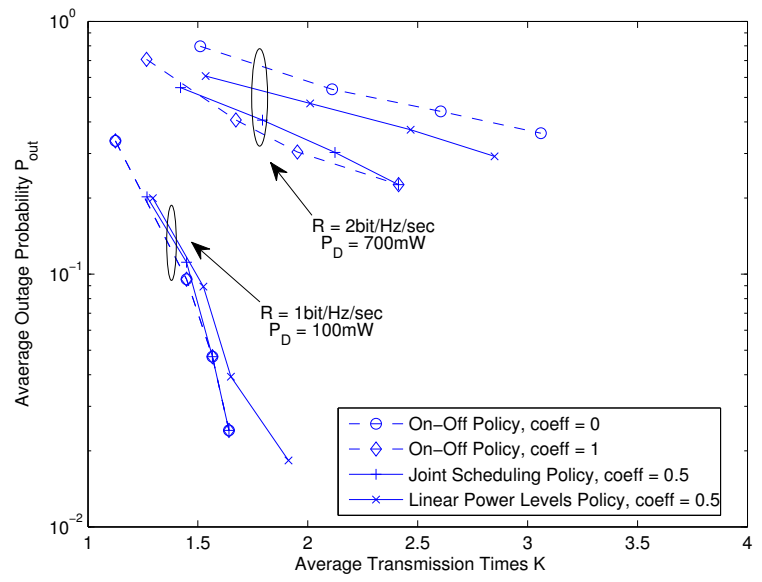

Fig. 6. The tradeoff between the minimum $P_{\text {out }}$ and the corresponding average transmission time $\tau$, with different maximum transmission times $K$. The representative system parameters chosen are $R=2 \mathrm{bit} / \mathrm{Hz} / \mathrm{s}, P_{D}=$ $700 \mathrm{~mW}$, and $R=1 \mathrm{bit} / \mathrm{Hz} / \mathrm{s}, P_{D}=100 \mathrm{~mW}$.

in stationary Rayleigh fading channel. Both the transmitter and the receiver are with finite capacity of energy buffer, and the correlation between the energy arrivals at both nodes are considered. We focused on the outage minimization problem for various scenarios with different system parameters. We analytically calculate the thresholds and compare them with the results generated by a local searching algorithm based on the FSMC. We also proved that under the threshold-based On-Off policy, the optimal transmit power is related with the correlation between the energy arrivals of both nodes. Under the joint scheduling policy, the optimal transmit power is independent with the correlations between energy arrivals. Our numerical results show the performances of these policies, as well as the tradeoff between the average outage probability and average retransmission times.

\section{ACKNOWLEDGEMENT}

This work is sponsored in part by the National Science Foundation of China (NSFC) under grant No. 61201191, the National Basic Research Program of China (973 Program: 2012CB316001), the NSFC Excellent Young Investigator Award No. 61322111, the Creative Research Groups of NSFC under grant No. 61321061, MoE new century talent program No. NCET-12-0302, Beijing Nova Program No. Z121101002512051, and Hitachi R\&D Headquarter.

\section{APPENDIX}

\section{PROOF OF PROPOSITION 2}

First, consider the case of $\lambda_{e} \geq \max \left(P_{S}, P_{D}\right)$, it can be easily obtained that $\Psi\left(P_{S}, P_{D}\right)=1$ by extending the proof of Theorem 1 in ref. [10]. Next, consider the case of $\lambda_{e}<\max \left(P_{S}, P_{D}\right)$, we have $(\beta=S, D)$

$\sum_{t=1}^{n} \mathbf{E}\left[E_{\beta}^{t}\right]=P_{\beta} \sum_{t=1}^{n} \mathbf{E}\left[I\left(B_{S}^{t} \geq P_{S}, B_{D}^{t} \geq P_{D}\right)\right]+\mathbf{E}\left[B_{\beta}^{n}\right]$
Since $\mathbf{E}\left[B_{\beta}^{t}\right] \geq 0$, we have

$$
\begin{aligned}
\lambda_{e} & \geq P_{\beta} \lim _{n \rightarrow \infty} \frac{1}{n}\left\{\sum_{t=1}^{n} \mathbf{E}\left[I\left(B_{S}^{t} \geq P_{S}, B_{D}^{t} \geq P_{D}\right)\right]\right\} \\
& =P_{\beta} \Psi\left(P_{S}, P_{D}\right),
\end{aligned}
$$

For $\forall t$ and $B_{\beta}^{0}=0$, from Eq. (8) we have $\frac{1}{P_{S}}\left(B_{S}^{t}-B_{S}^{t+1}+\right.$ $\left.E_{S}^{t}\right)=\frac{1}{P_{D}}\left(B_{D}^{t}-B_{D}^{t+1}+E_{D}^{t}\right)$. By taking the summation and expectation at both sides we obtain (assume $P_{S} \geq P_{D}$ )

$$
\lim _{n \rightarrow \infty} \frac{1}{n} \sum_{t=1}^{n} \mathbf{E}\left[B_{S}^{n}\right] \leq \frac{P_{S}}{P_{D}}\left(\lim _{n \rightarrow \infty} \frac{1}{n} \sum_{t=1}^{n} \mathbf{E}\left[B_{D}^{n}\right]\right) .
$$

For battery state $B_{S}^{t}$, by applying (29) we can get

$$
\begin{aligned}
\lambda_{e} & \leq \lim _{n \rightarrow \infty} \frac{P_{S}}{n}\left\{\sum_{t=1}^{n} \mathbf{E}\left[I\left(B_{S}^{t} \geq P_{S}, B_{D}^{t} \geq P_{D}\right)\right]+\frac{\mathbf{E}\left[B_{D}^{n}\right]}{P_{D}}\right\} \\
& =P_{S} \Psi\left(P_{S}, P_{D}\right),
\end{aligned}
$$

and thus we obtain $\Psi\left(P_{S}, P_{D}\right) \geq \frac{\lambda_{e}}{P_{S}}$. Combining (27) and (30) we complete the proof of the proposition.

\section{REFERENCES}

[1] V. Sharma, U. Mukherji, V. Joseph, and S. Gupta, "Optimal Energy Management Policies for Energy Harvesting Sensor Nodes," IEEE Trans. Wireless Commun., vol. 9, no. 4, pp. 1326-1336, Apr. 2010.

[2] C. Huang, R. Zhang, S. Cui, "Outage Minimization in Fading Channels under Energy Harvesting Constraints," in Proc. ICC Workshop on Energy Harvesting for Communications, June 2012.

[3] O. Ozel, K. Tutuncuoglu, J. Yang, S. Ulukus, and A. Yener, "Transmission with Energy Harvesting Nodes in Fading Wireless Channels: Optimal Policies", IEEE J. Sel. Areas Commun., vol. 29, no. 8, pp. 1732-1743,

[4] I. Krikidis, T. Charalambous, and J. S. Thompson, "Stability Analysis and Power Optimization for Energy Harvesting Cooperative Networks," IEEE Signal Processing Letters, vol. 19, no. 1, pp. 20-23, Jan. 2012.

[5] A. Aprem, C. R. Murthy, N. B. Mehta, "Transmit Power Control Policies for Energy Harvesting Sensors with Retransmissions," IEEE Journal of Selected Topics in Signal Processing, vol. 7, no. 5, pp. 895-906, Oct. 2013. Sept. 2011.

[6] B. Medepally, N. B. Mehta, C. R. Murthy, "Implications of Energy Profile and Storage on Energy Harvesting Sensor Link Performance", in IEEE Global Telecom. Conf., Dec. 2012.

[7] S. Cui, A. J. Goldsmith, and A. Bahai, "Energy-efficiency of MIMO and Cooperative MIMO Techniques in Sensor Networks," IEEE J. Sel. Areas Commun., vol. 22, no. 6, pp. 1089-1098, Aug. 2004.

[8] D. Tse, Fundamentals of Wireless Communication, Cambridge Univ, Press, 2005.

[9] P. Grover, K.A. Woyach, and A. Sahai, "Towards a Communicationtheoretic Understanding of System-level Power Consumption", IEEE J. Sel. Areas Commun., vol. 29, no. 8, pp. 1744-1755, Aug. 2011.

[10] K. Huang, "Spatial Throughput of Mobile Ad Hoc Network with Energy Harvesting", IEEE Trans. on Information Theory, vol. 59, no. 11, pp. 7597-7612, Nov. 2013.

[11] B. Berglund, J. Johansson, and T. Lejon, "High Efficiency Power Amplifiers", Ericsson, Mar. 2006.

[12] S. Zhou, T. Chen, W. Chen, Z. Niu, "Outage Minimization for a Fading Wireless Link with Energy Harvesting Transmitter and Receiver", in preparation. 